\title{
La aplicación de un Ciclo de Mejora Docente en la enseñanza de la Paleografía en las aulas universitarias
}

\author{
ADRIÁN ARES LEGASPI \\ Universidad de Sevilla \\ Departamento de Historia Medieval y \\ Ciencias y Técnicas Historiográficas \\ aares@us.es \\ ORCID: https://orcid.org/0000-0003-0241-4883 \\ D.O.I.: http://dx.doi.org/10.12795/JDU.2018.i01.73 \\ Pp.: 1307-1323
}

\section{Resumen}

Presentamos los resultados de un Ciclo de Mejora Docente (CMD) de 6 horas aplicado a la enseñanza universitaria de la escritura gótica, abarcando 3 aspectos de esta práctica: contenidos, metodología de enseñanza y evaluación. Ciclo con unos resultados satisfactorios y unos efectos positivos en la evolución del aprendizaje de los estudiantes.

Palabras clave: Paleografía, Grado en Historia, Docencia Universitaria, Experimentación Docente Universitaria 


\section{Introducción}

Han sido diversos los investigadores que desde el campo de las ciencias y técnicas historiográficas han abordado la cuestión de su enseñanza en las aulas universitarias (Cárcel Ortí, 2002; Ares Legaspi y Belmonte Fernández, 2017). Ya que estas consideran la fuente histórica primaria como el principal objeto de estudio, los contenidos desarrollados para su aprendizaje deberán aunar el aspecto conceptual y procedimental.

El CMD se aplica al tema "Escritura gótica" (al impartirse a mediados del cuatrimestre los alumnos ya conocen la metodología paleográfica) de la asignatura Paleografía (Grado de Historia de la Universidad de Sevilla), de tercer curso (los estudiantes ya poseen una base de conocimientos amplia y el nivel de formulación inicial de los contenidos es medio) y en el grupo de tarde, con una asistencia de 30 alumnos. En ella se ensaña la historia de la escritura latina (sobre todo en la Corona de Castilla) desde época romana hasta los siglos XVI-XVII. Otro CMD en el área de la Historia puede verse en Fuentes Barragán, 2017.

\section{Diseño del CMD: contenidos, metodología y actividades}

La finalidad del CMD es que los estudiantes conozcan y comprendan las características internas y externas de la escritura gótica. Además, aprenderán a leer documentos de ese período e identificar, describir, clasificar y analizar críticamente sus elementos gráficos.

La materia consta de dos partes (Figura 1), una dedicada a los contenidos conceptuales y otra a los procedimentales. En la primera se estudia la escritura gótica como fenómeno social e histórico, desde 3 perspectivas: a) conjunto de formas creadas por el hombre (qué tipo de escritura y cuáles son sus características formales nos ayudará 
a su posterior identificación y clasificación); b) producción con una historia propia (dónde se practica, por quién o con qué fin permitirán el estudio social de la escritura); y, c) elemento de una estructura mayor que es la civilización de una época (cómo influyen otros factores del contexto sobre la escritura refleja la complejidad del fenómeno).

Los contenidos procedimentales comprenden 2 tareas interdependientes: lectura de la escritura gótica y su análisis práctico. Los alumnos dominarán la lectura de textos en castellano (SS. XIII-XVI), realizados con distintos modelos de escritura gótica; aprenderán cómo identificarlos, cómo describir sus características y cómo clasificarlos o relacionarlos con otros componentes de la sociedad del momento. Esto permite no solo estudiar la escritura como objeto de trabajo independiente sino también desarrollar la crítica de fuentes históricas.

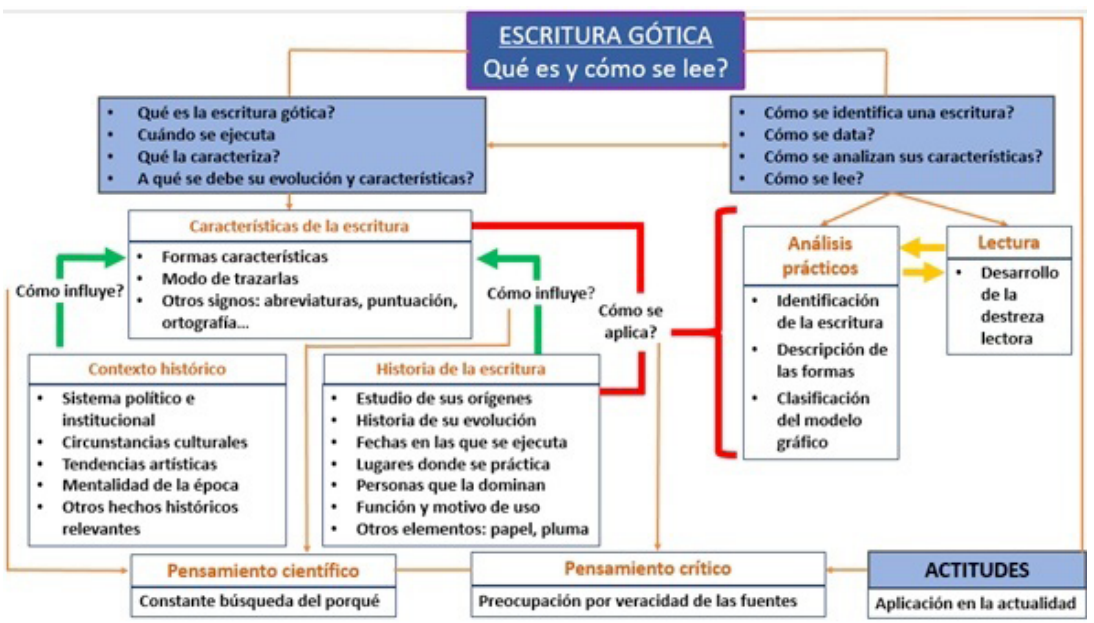

Figura 1. Mapa de contenidos

El modelo metodológico se divide en 3 partes, con el fin de establecer un aprendizaje lo más completo posible, seguro y de manera asistida por parte del profesor y con gran peso de la actividad del alumno. Este esquema (Figura 2) será seguido tanto en las 3 sesiones dedicadas a los contenidos conceptuales como a las 3 destinadas a los procedimentales. 
Después de una somera introducción sobre el tema del CMD y un repaso de la materia explicada en las sesiones anteriores, se pasará a los alumnos el cuestionario para, como luego veremos, evaluar sus ideas iniciales sobre la escritura gótica.

El tercer paso es el planteamiento de un problema que iniciará cada parte de los contenidos y que los alumnos resolverán en grupo, siendo la participación del profesor menor. Se pretende generar un debate entre profesor y estudiantes al hilo del problema, al cual el docente añadirá cuestiones que lo enriquezcan y hagan más complejo, con el fin de que el alumno comience a construir el aprendizaje razonada y críticamente dentro de una "atmósfera interactiva" que fomente su confianza y evite el temor a la equivocación (Bain, 2007).

El cuarto paso es la explicación teórica de los contenidos conceptuales por el docente, que serán trabajados en clase mediante preguntas entre profesor y alumnos. No obstante, el papel del docente aumenta respecto a la fase anterior, creciendo también el contenido conceptual.

Tras ello, la participación de los estudiantes aumenta al pasar a una etapa de actividades prácticas. Con ello se pretende asentar los conceptos aprendidos y aplicarlos a un caso práctico a modo de recapitulación. El docente ocupa ahora una posición menos participativa para que los alumnos construyan el conocimiento por ellos mismos (Finkel, 2008).

Por último, se pasará de nuevo el cuestionario inicial de evaluación y se sumará una mención a la bibliografía sobre la escritura gótica y una conclusión al tema aportada por el profesor; mientras que los alumnos podrán añadir las principales ideas que han aprendido. 
I: Introducción [C: Comentario de lo que se explicará en la sesión; R: Repaso breve de lo visto en la clase anterior]

IA: Ideas iniciales de los alumnos

PB: Problema inicial

T: Teoría

PR: Práctica

EA: Evaluación ideas de los alumnos

C: Conclusión

A: Alumnos

P: Profesor

Figura 2. Modelo Metodológico.

Para llevar a cabo el CMD, se diseñó una serie de actividades (Tabla 1). También se diseñó un cuestionario inicial con el que evaluar las ideas previas y finales de los estudiantes sobre la escritura gótica:

a) ¿Cómo definirías la escritura gótica?

b) ¿Cuándo o durante qué período temporal se desarrolló este modelo gráfico en Europa? ¿Crees que este desarrollo ha sido similar a lo largo de todos los territorios del occidente europeo, o bien, diferente según la zona? De ser así, ¿cuál sería la cronología en el caso de la Península Ibérica?

c) ¿Cuáles son los principales elementos que la caracterizan desde el punto de vista de su morfología y el trazado de las letras?

d) Teniendo en cuenta los distintos ámbitos de uso de una escritura que hemos visto en los temas anteriores y los productos escritos elaborados por una sociedad, ¿crees que existía una única variedad de escritura gótica o más de una? En este caso, ¿cuáles serían esos modelos diversos dentro de la escritura gótica? 


\section{Tabla 1}

Secuencia de actividades.

\begin{tabular}{|c|l|c|}
\hline $\begin{array}{c}\text { Tipo de actividad } \\
\text { Fase del modelo } \\
\text { metodológico }\end{array}$ & \multicolumn{1}{|c|}{ Descripción } & Sesión 1 \\
\hline \multicolumn{3}{|c|}{ Temporalización } \\
\hline Introducción & $\begin{array}{l}\text { Introducción a los contenidos de } \\
\text { las sesiones y recapitulación de } \\
\text { la anterior }\end{array}$ & 5 minutos \\
\hline Ideas previas del & $\begin{array}{l}\text { Recogida de ideas de los } \\
\text { estudiantes }\end{array}$ & 15 minutos \\
\hline alumno & $\begin{array}{l}\text { Problema inicial y comentario crí- } \\
\text { tico de un texto sobre historia de } \\
\text { la escritura; preguntas del profe- } \\
\text { sor a los alumnos y debate de las } \\
\text { mismas }\end{array}$ & S0 minutos \\
\hline \multicolumn{3}{|c|}{ Sesión 2 } \\
\hline Problema inicialón 3
\end{tabular}

Jornadas de Formación e Innovación Docente del Profesorado I № 1 (2018) Esta obra se distribuye con la licencia Creative Commons Reconocimiento-NoComercial-SinObraDerivada Internacional (CC BY-NC-ND 4.0.) 


\begin{tabular}{|c|c|c|}
\hline Teoría & $\begin{array}{l}\text { Explicación oral y mediante } \\
\text { PowerPoint por el profesor de } \\
\text { cómo leer documentos, con qué } \\
\text { técnicas y elementos paleográfi- } \\
\text { cos de la escritura }\end{array}$ & 30 minutos \\
\hline Práctica & $\begin{array}{l}\text { Toda la clase leerá y transcribirá } \\
\text { un mismo documento de manera } \\
\text { de forma individual }\end{array}$ & 30 minutos \\
\hline \multicolumn{3}{|c|}{ Sesión 6} \\
\hline Práctica & $\begin{array}{l}\text { Los alumnos compararán en } \\
\text { grupo sus transcripciones y anali- } \\
\text { zarán la escritura }\end{array}$ & 15 minutos \\
\hline Práctica & $\begin{array}{l}\text { Cada grupo leerá en alto parte } \\
\text { del documento y expondrá su } \\
\text { análisis para que el profesor lo } \\
\text { corrija y realice cuestiones en } \\
\text { alto al resto de la clase }\end{array}$ & 25 minutos \\
\hline $\begin{array}{c}\text { Evaluación ideas } \\
\text { de los alumnos }\end{array}$ & $\begin{array}{l}\text { Los estudiantes realizarán de } \\
\text { nuevo el cuestionario inicial uti- } \\
\text { lizado para evaluar sus ideas } \\
\text { previas y así poder evaluar su } \\
\text { aprendizaje }\end{array}$ & 10 minutos \\
\hline Conclusión & $\begin{array}{l}\text { Recapitulación del profesor y } \\
\text { principales ideas de los alumnos. } \\
\text { Bibliografía del tema }\end{array}$ & 10 minutos \\
\hline
\end{tabular}

\section{Desarrollo de las actividades}

En la primera sesión se recogieron las ideas previas de los alumnos y se realizaron varios comentarios críticos y debate sobre textos científicos de diversa temática: estudios paleográficos de caso, textos historiográficos de la disciplina o algunos de otras ciencias que tratan la escritura desde una perspectiva distinta. Empezamos con un estudio sobre las teorías de Tomás de Aquino, lo cual desconcertó a gran parte de los alumnos, pero era el efecto que se buscaba para atraer su atención. Tras realizar varias 
cuestiones sobre el texto, a las que contestaron en torno a un tercio de la clase (11 o 12), el tema se orientó hacia la escritura gótica preguntándoles si creían que el pensamiento escolástico había influido sobre la cultura escrita de la época, cómo y por qué. Esto dio paso al comentario crítico de textos relacionados con la historia de la escritura y que sirvió para avanzar los contenidos conceptuales de la siguiente sesión: a) un texto sobre tipologías documentales; b) otro sobre escribiente, y c) un último sobre el contexto histórico. El número de alumnos que participó se mantuvo similar, mientras que el docente se limitaba a guiar el debate mediante preguntas a los estudiantes.

La segunda sesión se dedicó exclusivamente a la exposición oral por el profesor de los contenidos conceptuales (historia de la escritura, características e influencias del contexto histórico, etc.), usando otras herramientas como PowerPoint, imágenes de documentos y libros... En este sentido, la sesión inicial del CMD hizo que los alumnos poseyesen una base de conocimientos de estos contenidos, lo cual permitió que, al hilo de las explicaciones, se realizasen constantes referencias a lo explicado anteriormente, planteando preguntas y breves discusiones sobre ciertas ideas, sin tener que obligar a los estudiantes a responder, pues ellos mismos se animaban a hacerlo. Se estableció, por tanto, un ambiente de reciprocidad.

En la sesión 3 se realizaron comentarios de textos o de teorías de otros paleógrafos, pero ahora con los conocimientos adquiridos anteriormente. Los alumnos trabajaron sobre casos concretos: un texto sobre el contexto histórico de la escritura gótica, un texto sobre las características de la escritura, y un cuadro de clasificación de sus tipos y nomenclaturas. En este caso la intervención del docente fue mucho menor con el fin de que el peso de la clase recayese sobre los alumnos, generándose un debate de ideas para rebatir, comparar y analizar los textos. 
Intercambio de ideas entre de nuevo un tercio de la clase, que muchas veces se limitó a su exposición por los alumnos, sin llegar a un análisis y una comparación profunda.

La sesión 4 se dedicó a los contenidos procedimentales: cómo leer la escritura gótica. Se inició con un problema al que responder entre todos mediante una serie de preguntas y al que se añadieron otras imágenes o comentarios para contraponer las características de otras letras. Los alumnos analizaron durante unos minutos un documento del siglo XIII (para lo cual debieron empezar su lectura), para luego responder en alto a dos preguntas: qué tipo de letra era y si era rápida o lenta. En este primer instante, el número de alumnos que respondieron descendió respecto a las sesiones anteriores, posiblemente debido al conocimiento más técnico y especializado que implicaban estas cuestiones, creciendo el miedo de los estudiantes a fallar. La sesión continuó con más cuestiones (tipos de abreviaturas y ligaduras, si era una escritura elegante o descuidada...). Además, los estudiantes fueron exponiendo sus dudas, para cuya respuesta usamos la pizarra para dibujar la forma y trazado de las letras.

En la primera parte de la sesión 5 se explicaron los contenidos conceptuales relativos a la lectura de documentos (abreviaturas, tipos de letras o técnicas de lectura y transcripción). Ahora bien, el carácter técnico de estos contenidos supuso una gran dificultad para los alumnos, ya que en diversas ocasiones manifestaron la complejidad para entender ciertos conceptos. Dificultades que quedaron patentes en la segunda mitad de la sesión, la destinada a la lectura individual de los documentos por los estudiantes, pues no todos ellos fueron capaces de leerlos, planteando muchas preguntas sobre esta tarea y el análisis paleográfico del texto.

En la última sesión, los alumnos compararon en grupos sus transcripciones y comentaron sus características 
paleográficas. Tras ello, cada grupo realizó una lectura de los textos en alto para, esta vez, corregir errores entre todos o responder a las preguntas que el profesor planteaba al hilo de las transcripciones (contenido de palabras, normas de transcripción u otros elementos paleográficos). Es por ello que la participación aumentó de nuevo debido al componente de obligatoriedad que requería la actividad.

\section{Evaluación del aprendizaje}

De los 35 alumnos que realizaron el primer cuestionario sobre sus ideas iniciales, solo 18 asistieron el día que se pasó el cuestionario final, por lo que los porcentajes se calculan sobre este total. Los resultados se representan mediante escaleras de aprendizaje (ver Figuras 3 a 6), donde se incluye el tipo de respuesta y los obstáculos para evolucionar a un tipo superior.

En general, el proceso de aprendizaje de los alumnos fue satisfactorio. Como reflejan las escaleras, se produce entre los estudiantes una tendencia a mejorar los niveles de análisis y conocimiento de la materia respecto a la situación inicial, adquiriendo unos conocimientos conceptuales y procedimentales muy técnicos y complejos. Así, son los porcentajes de los niveles superiores en el tipo de respuestas, aquellos donde el alumno relaciona varios aspectos de la escritura (sus formas, contexto histórico o funciones y tipologías), los que más crecen en el cuestionario final respecto al inicial, donde los niveles inferiores poseían mayor peso.

Por otra parte, hay que tener en cuenta que esta jerarquía en el nivel de respuestas también se correspondía con una jerarquía en el tipo de preguntas. Las dos primeras cuestiones más abiertas, por lo que daban mayor margen al alumno para especular con su solución. Por el contrario, las dos últimas preguntas requerían un mayor conocimiento de esta actividad humana y, sobre todo, un 
razonamiento más complejo y "multicausal". Con esta diversidad de cuestiones pretendíamos que el alumno se enfrentase a preguntas que, dadas en ese orden, requerían cada vez una mayor destreza analítica y que por lo tanto el estudiante modificase su forma de reflexionar sobre la escritura y la manera en que afronta su análisis.



Figura 3. Escalera de aprendizaje correspondiente a la pregunta A.

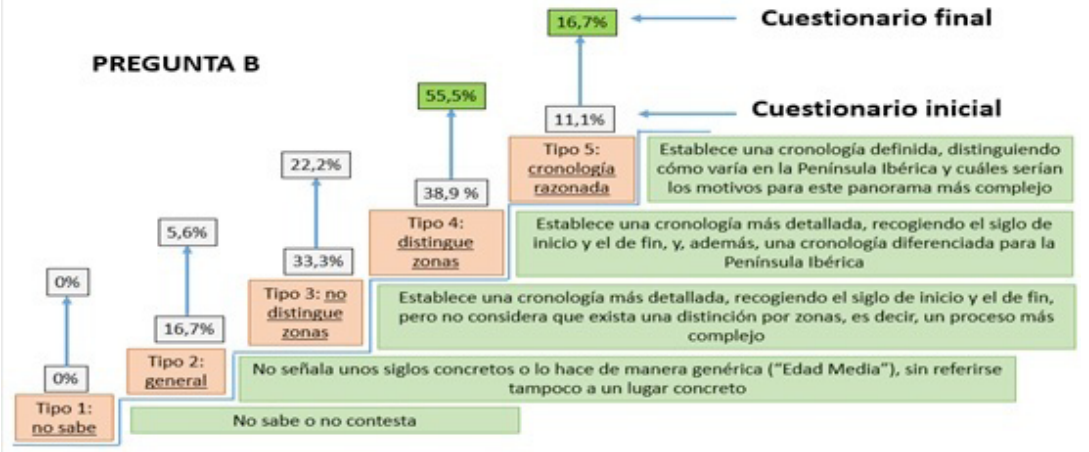

Figura 4. Escalera de aprendizaje correspondiente a la pregunta B.



Figura 5. Escalera de aprendizaje correspondiente a la pregunta C.

Jornadas de Formación e Innovación Docente del Profesorado | № 1 (2018)

(c) Esta obra se distribuye con la licencia Creative Commons Reconocimiento-NoComercial-SinObraDerivada $\quad 4.0$ Internacional (CC BY-NC-ND 4.0.) 


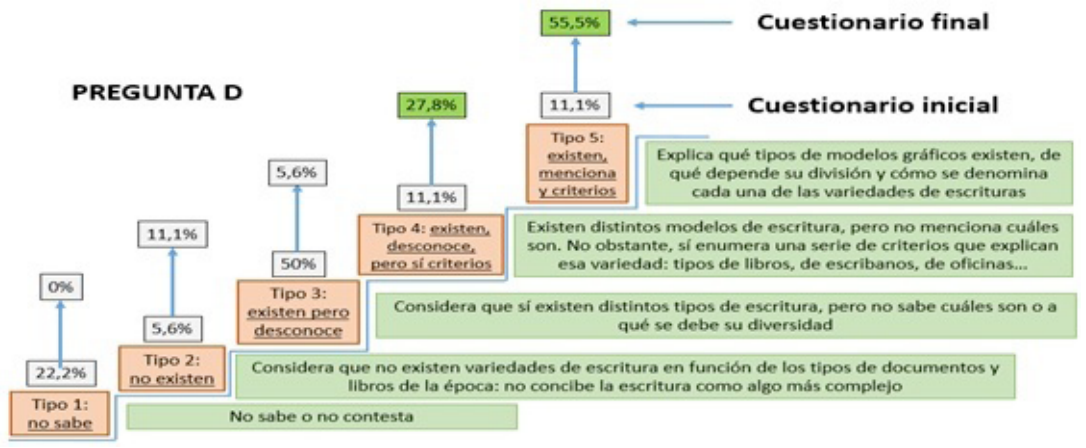

Figura 6. Escalera de aprendizaje correspondiente a la pregunta D.

En la Figura 7 podemos observar con más detalle la evolución de cada alumno, comprobando dónde se produjeron los principales avances en el aprendizaje o dónde encontraron más dificultades. Los datos muestran en general una evolución positiva en todos los estudiantes. Sin embargo, en algunos casos el nivel de las respuestas de un mismo alumno es idéntico en ambas fases del aprendizaje o incluso inferior al final. En nuestra opinión, no se debe hablar de un empeoramiento en el aprendizaje, sino de un cambio en la perspectiva del alumno a la hora de contestar. No es casualidad que este mantenimiento de la idea inicial o el descenso en el nivel de la respuesta se produzca en las cuestiones más abiertas, ya que su carácter pudo llevar a esos alumnos a contestar creando hipótesis abiertas. Es decir, dentro de estas ideas contemplaban más factores que los que luego expresan en el cuestionario final, cuando, tras las 6 sesiones del CMD, contestan con datos más concretos.

Esta dinámica se opone a la percibida en las dos últimas preguntas, donde la evolución del aprendizaje tiende claramente a la mejora, a respuestas más complejas. Como se trata de cuestiones técnicas y específicas de la disciplina, es muy difícil que el alumno cuente con un bagaje inicial en cuanto a las características o variedades de la escritura gótica. Algunos de ellos sí demuestran poseer 
ciertos rudimentos (no descartamos que puedan ser repetidores) que mantienen desde el principio del ciclo de mejora (alumnos 1 y 4 en la última pregunta y el 8 en la tercera pregunta); pero la mayoría responden al cuestionario inicial en términos generales o reflejando su desconocimiento. Frente a esto, tras las 6 sesiones, la mayoría de los estudiantes mejoraron su conocimiento sobre estos aspectos de la escritura gótica, predominando los niveles más altos tanto en la tercera como en la cuarta pregunta.

A pesar de no considerar como un retroceso en el aprendizaje los "casos negativos" de algunas respuestas en las dos primeras cuestiones, no descartamos que en algunos casos sí pudiese operar un desconocimiento de los contenidos, es decir, un fallo en el proceso de aprendizaje. El hecho de no alcanzar los objetivos en este punto es, en nuestra opinión, a la vez sintoma y consecuencia de las dos principales dificultades que hemos encontrado en la puesta en práctica de este CMD: el número de sesiones y la diversidad de intereses y motivaciones de los estudiantes. Por un lado, creo que para la correcta enseñanza de un tema tan amplio como la escritura gótica lo ideal sería contar con, como mínimo, el doble de sesiones para poder profundizar en todos los contenidos de la materia. Por otro lado, en los grupos de las tardes suele haber un gran número de alumnos que no realizan el grado con el fin de dedicarse profesionalmente a lo que estudian o que no desean continuar por la vía de la investigación. Esto hace que en una misma aula convivan intereses, motivaciones, objetivos y necesidades por parte de los alumnos de muy diferente índole, dando lugar a una situación de múltiples y complejos "estilos de aprendizaje" (Giné Freixes, 2009). Es por ello que las actividades que tenemos que manejar deberían ser todavía más diversas y estimulantes (Alba y Porlán, 2017) y capaces de cubrir todas estas exigencias. En definitiva, la posible ineficacia en el aprendizaje que muestran algunos de los cuestionarios individuales podría 
solventarse con el cambio de actividades realizadas en la clase, intentando buscar otro tipo de ejercicios que se ajusten más a las necesidades de esos alumnos, sin trastocar por ello la evolución positiva -y mayoritaria- en el aprendizaje del resto de los estudiantes.

\begin{tabular}{|c|c|c|c|c|c|c|c|c|}
\hline & $\begin{array}{c}\text { CUESTIONARIO } \\
\text { INICIAL. }\end{array}$ & $\begin{array}{c}\text { CUESTIONARIO } \\
\text { FNAL }\end{array}$ & $\begin{array}{c}\text { CUESTIONARIO } \\
\text { INICIAL }\end{array}$ & $\begin{array}{c}\text { CUESTIONARIO } \\
\text { FINAL }\end{array}$ & $\begin{array}{c}\text { CUESTIONARIO } \\
\text { INICIAL. } \\
\end{array}$ & $\begin{array}{l}\text { CUESTIONARIO } \\
\text { FINALL }\end{array}$ & $\begin{array}{l}\text { CUESTIONARIO } \\
\text { INICIAL. }\end{array}$ & $\begin{array}{c}\text { CUESTIONARIO } \\
\text { FINAL }\end{array}$ \\
\hline & PREGUNTAA & PREGUNTAA A & PREGUNTA B & PREGUNTA B & PREGUNTAC & PREGUNTAC & PREGUNTAD & PREGUNTA D \\
\hline & Niveles 1.5 & Niveles 1.5 & Niveles 1-5 & Niveles 1.5 & Niveles $1-4$ & Niveles $1-4$ & Niveles 1-5 & Niveles 1.5 \\
\hline Alumno 1 & 4 & 3 & 4 & 3 & 1 & 2 & 5 & 5 \\
\hline Alumno 2 & 3 & 1 & 4 & 5 & 2 & 2 & 2 & 4 \\
\hline Alumno 3 & 5 & 5 & 3 & 4 & 4 & 3 & 3 & 3 \\
\hline Alumno 4 & 3 & 5 & 5 & 4 & 2 & 2 & 5 & 5 \\
\hline Alumno 5 & 3 & 3 & 3 & 3 & 1 & 2 & 1 & 2 \\
\hline Alumno 6 & 2 & 5 & 4 & 4 & 1 & 3 & 3 & 2 \\
\hline Alumno 7 & 5 & 3 & 3 & 5 & 3 & 3 & 4 & 5 \\
\hline Alumno 8 & 1 & 1 & 2 & 4 & 4 & 4 & 1 & 5 \\
\hline Alumno 9 & 4 & 5 & 5 & 4 & 1 & 4 & 1 & 5 \\
\hline Alumno 10 & 1 & 3 & 5 & 3 & 1 & 2 & 4 & 5 \\
\hline Alumno 11 & 3 & 4 & 2 & 2 & 2 & 3 & 1 & 4 \\
\hline Alumno 12 & 4 & 5 & 4 & 4 & 3 & 3 & 3 & 5 \\
\hline Alumno 13 & 2 & 2 & 3 & 4 & 2 & 3 & 3 & 4 \\
\hline Alumno 14 & 2 & 5 & 4 & 4 & 1 & 4 & 3 & 5 \\
\hline Alumno 15 & 3 & 5 & 4 & 5 & 1 & 3 & 3 & 5 \\
\hline Alumno 16 & 3 & 5 & 3 & 4 & 1 & 3 & 3 & 5 \\
\hline Alumno 17 & 2 & 5 & 2 & 4 & 1 & 4 & 3 & 4 \\
\hline Alumno 18 & 2 & 2 & 3 & 3 & 2 & 3 & 3 & 4 \\
\hline
\end{tabular}

Figura 7. Evolución del aprendizaje por estudiante.

\section{Evaluación del ciclo de mejora docente}

La evaluación del CMD ha sido realizada en conjunto por profesor y alumnos, pues se les ha solicitado que expliquen por escrito las principales ideas que retenían después de estas 6 sesiones y que aportasen también su opinión sobre la metodología y actividades desarrolladas.

En lo referente a los contenidos de la materia, la mayoría de los alumnos destacan ideas de calado, considerando igual de importante las cuestiones conceptuales y las procedimentales. En nuestra opinión, mantener este equilibrio entre ambas partes es fundamental en el éxito del aprendizaje de los alumnos. Un equilibrio evidenciado en los buenos resultados del CMD, pero que podría ser más eficaz si conseguimos cambiar las actividades propuestas. Aumentar la naturaleza y variedad de las tareas podría adecuar mejor los distintos estilos de aprendizaje a las necesidades de cada estudiante. En este sentido, la 
entrega por escrito al docente de los ejercicios realizados en las sesiones podría ayudarnos a captar las condiciones de cada uno de esos estilos y a adaptar dichas actividades.

En cuanto a la metodología de enseñanza, los alumnos muestran un alto grado de satisfacción con la participación en las sesiones. Muchos consideran que los comentarios de textos en alta voz o mediante preguntas planteadas por el docente es una fórmula de impartición de la materia muy adecuada para los fines perseguidos. De hecho, ellos mismos son conscientes de sus beneficios, reconociendo la necesidad de un proceso de aprendizaje crítico y la comprensión razonada ("resulta más interesante a veces comprender el origen de cada escritura que la propia práctica"). Mantener esta interacción entre profesor (como guía del pensamiento analítico de los alumnos) y estudiantes es crucial para generar un aprendizaje construido por los alumnos.

No obstante, muchas críticas se centraron en el desarrollo continuo de actividades, proponiendo algunos estudiantes que se obligue a "hacer en casa alguna lámina para transcribir", recalcando otros la importancia de los ejercicios "para ir viendo la evolución personal sin necesidad de evaluar". Esto demuestra la buena acogida de esas actividades y la motivación del alumnado y la necesidad por nuestra parte de incorporar -debido al carácter progresivo y evolutivo del aprendizaje (Rivero y Porlán, 2017)- una evaluación continua de los conocimientos de los estudiantes con ejercicios que, aun no contabilizando en la calificación final, les permitan a ellos mismos comprobar sus conocimientos y niveles de aprendizaje.

Por último, teniendo en cuenta estas consideraciones, podríamos resumir nuestros principios didácticos en los siguientes puntos:

a) Para un correcto aprendizaje este se producirá en un contexto de reciprocidad entre profesor y alumnos, fomentando el primero un ambiente de confianza y estimulante para la participación de los segundos. 
b) El aprendizaje deberá ser construido por el alumno en la medida de lo posible, generándose una adquisición del conocimiento por su parte de manera activa y desarrollando su pensamiento crítico y racional.

c) Para lograr este proceso de aprendizaje es necesario fomentar la motivación intrínseca del estudiante y conseguir una realimentación constante de sus intereses en la materia.

d) El profesor debe alcanzar una posición equilibrada entre la figura de transmisor de contenidos y de guía que orienta un aprendizaje construido por el alumno.

e) Los contenidos deben ser lo más contrastados y actualizados posible, abarcando de manera integral aspectos conceptuales, procedimentales y los valores de la disciplina.

f) La metodología de la enseñanza combinará la actuación del profesor como emisor de contenidos y la participación activa y constante de los alumnos, adquiriendo estos los conocimientos mediante una dinámica de retroalimentación.

g) Las actividades propuestas se ajustarán en lo posible a las necesidades y punto de partida de los alumnos y serán fácilmente modificables para adaptarse a la evolución del aprendizaje de los estudiantes.

h) La evaluación del aprendizaje de los alumnos se puede realizar a través de distintos mecanismos, no teniendo por qué suponer una calificación numérica definitiva. Esta evaluación tiene como objetivo conocer las necesidades, dificultades y ventajas de cada estudiante en el proceso cognitivo para poder mejorarlo y ajustarlo en cualquier momento, asi como para fomentar la motivación intrínseca de los alumnos. 


\section{Bibliografía}

Alba, N. de y Porlán, R. (2017). La metodología de enseñanza. En Porlán (Coord.) Enseñanza universitaria: cómo mejorarla (pp. 37-53). Sevilla: Ediciones Morata.

Ares Legaspi, A. y Belmonte Fernández, D. (2018). Aplicación de las TIC y otros recursos en la enseñanza y difusión de las ciencias y técnicas historiográficas (CCyTTHH). En Chaves-Montero (Ed.) Las TIC como plataforma de teleformación e innovación educativa en las aulas (pp. 7594). Sevilla: Egregius.

Bain, K. (2007). Lo que hacen los mejores profesores de universidad. Valencia: Universitat de València.

Cárcel Ortí, Ma M. (2002). La paleografía y diplomática en las universidades españolas. Signo: revista de historia de la cultura escrita, 9, 39-106.

Finkel, D. (2008). Dar clase con la boca cerrada. Valencia: Universitat de València.

Fuentes Barragán, A. (2017). Un ciclo de mejora en Historia. En Porlán (Coord.) Enseñanza universitaria: cómo mejorarla (pp. 159-168). Sevilla: Ediciones Morata.

Giné Freixes, N. (2009), Cómo mejorar la docencia universitaria: el punto de vista del estudiantado, Revista Complutense de Educación, 20(1), 117-134.

Rivero, A. y Porlán, R. (2017). La evaluación en la enseñanza universitaria. En Porlán (Coord.) Enseñanza universitaria: cómo mejorarla (pp. 73-91). Sevilla: Ediciones Morata.

Jornadas de Formación e Innovación Docente del Profesorado | № 1 (2018) Esta obra se distribuye con la licencia Creative Commons 